\title{
Intraluminal Tubal Endometriosis Mimicking Hydrosalpinx: Report of an Unusual Case
}

\author{
Ozturk Ea, Ugur MGa , Aydın $\mathrm{A}^{\mathrm{b}}$, Balat $\mathrm{O}^{\mathrm{a}}$ and Kalaycı $\mathrm{H}^{\mathrm{a}}$ \\ ${ }^{a}$ Gaziantep University, Department of Obstetric and Gynecology \\ ${ }^{b}$ Gaziantep University, Department of Pathology
}

ABSTRACT

Endometriosis is an estrogen-dependent disease causing pelvic pain and infertility and there is still limited knowledge of the pathogenesis. Hydrosalpinx is commonly seen in a heterogeneous spectrum of pathologies of distal tubal occlusion. In general, collection of fluid in uterine tubes occur as the end stage of pyosalpinx or as the advenced stage of endometriosis with pelvic adhesion. In this report we present a very rarely seen case that is called intraluminal tubal endometriosis mimicking hydrosalpinx without pelvic adhesion in an infertile patient. The clinical features and the management of intraluminal tubal endometriosis case which is very rare cause of enlarged tuba uterina in an infertile patient discussed.

KEYWORDS: Endometriosis, hydrosalpinx

\section{INTRODUCTION}

Endometriosis is a disease associated with endometrial glands and stroma abnormally lining outside the uterus. In fallopian tube, endometriosis can be related to at least three different unrelated lesions. ${ }^{1}$ One of them is intraluminal endometriosis, unassociated with endometriosis elsewhere.

In this report we present an intraluminal tubal endometriosis mimicing hydrosalpinx in an infertile patient who undergone laparoscopic unilateral salphingectomy. The clinical features and management of hydrosalpinx in infertile patient with keeping endometriosis in mind are discussed.

\section{CASE REPORT}

A 31 year old female patient referred to our hospital complaining of secondary infertility and chronic pelvic pain. She had a history of a vaginal delivery 6 years ago. She had 2 abortions which were at 6 th and 8 th weeks of gestation. She has not used any contraception methods for about two years.

On speculum examination neither leucorrhea, mucopurulent endocervicitis nor similar other pathologies was observed. General physical examination was

Corresponding author;

Ebru Ozturk

Department of Obstetric and Gynaecology

Gaziantep University, Turkey

e-mail: ebruozturkarslan@yahoo.com also normal. Pelvic examination revealed an adnexial fullness at the right adnexa. A pelvic ultrasound scan showed evidence of hydrosalpinx which was approximately $37 \times 12 \mathrm{~mm}$ in diameter at the right adnexial region (Figure 1). The uterus and bilateral ovaries were evaluated as normal by ultrasound scan. We performed diagnostic laparascopy and diagnosed dilated tuba uterine mimicking hydrosalpinx at the right adnexa. The other fallopian tube and bilateral ovaries were seen normal. There was no sign of endometriosis on ovaries or pelvic peritoneum. There was no peritubal adhesion. We perfomed laparoscopic right salpingectomy. The patient was discharged on the first postoperative day without any complications. The pathology report revealed the intraluminal tubal endometriosis.

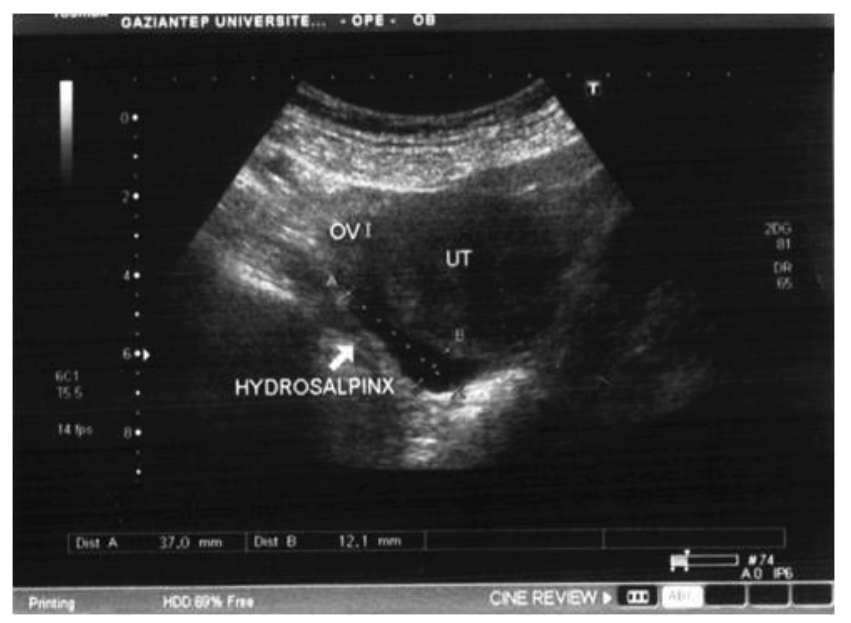

Figure 1. Ultrasonografic view of the case. There is a hipoechoic lesion in the right adnexia mimicking hyrosalpinx $(37 \times 12 \mathrm{~mm})$. 


\section{Pathology Report}

Microscopically, endometriotic foci were observed in the mucosa of fallopian tube. Eventhough all resection material was evaluated histologically, endometriotic focus only seen in the mucosa not in the serosa or myometrium of fallopian tube (Figure 2-3).

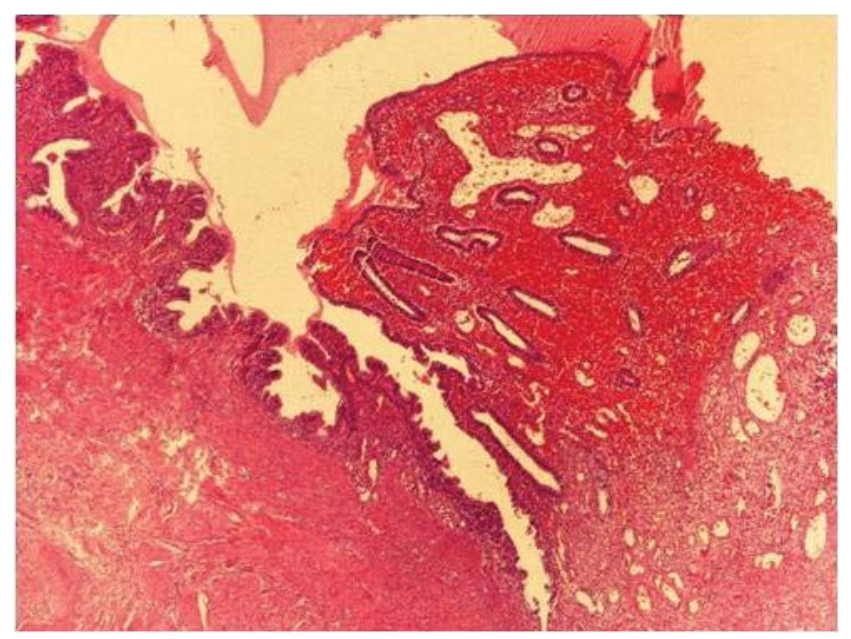

Figure 2. There is a endometriosis focus in the right region and epitelyum of fallopian tube in the left region ( $H \& E \times 40)$.

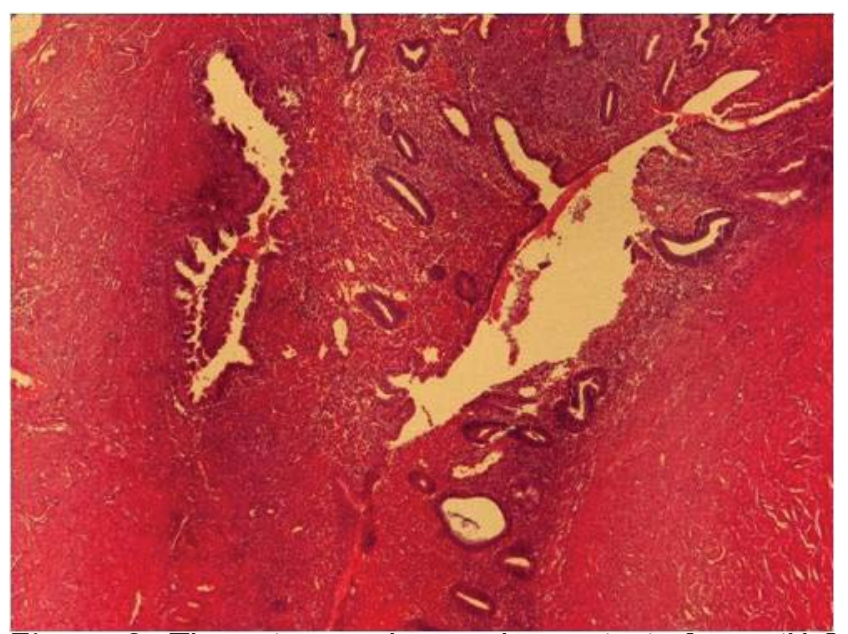

Figure 3. There is an other endometriosis focus ( $\mathrm{H} \&$ Ex 40).

\section{DISCUSSION}

Endometriosis is a disease associated with endometrial glands and stroma abnormally lining outside the uterus. Prevalence of endometriosis in reproductive age women is $3-10 \%$ and in infertile women $25-35 \%{ }^{2}$

Endometriosis can occur in almost every organ of the body and can be manifested different clinical symptoms mimicking unrelated diseases. For example endometriosis of the appendix can be seen as acute appendicitis or endometriosis can be manifested with acites as ovarian malignancy. ${ }^{3,4}$ In this report we present an intraluminal tubal endometriosis mimicing hydrosalpinx in an infertile patient.

Fallopian tube endometriosis can be classified to three different unrelated lesions in pathologic exami- nation. ${ }^{1}$ The most common type is serosal or subserosal endometriosis, typically related with endometriosis elsewhere in the pelvis without the myosalpinx. The second type of tubal endometriosis occurs in the tip of the proximal tubal stump, 1 to 4 years following tubal ligation which is called post salpingectomy endometriosis. ${ }^{5}$ The third and rare type is intraluminal endometriosis is typically unassociated with endometriosis elsewhere. ${ }^{1}$

In our patient we observed blood-filled fluid tubes, looks like chocolate cyst of ovaries which caused enlarged of tuba uterina without other endometriotic lesions. This is very rare presentation of endometriosis. Clinically some studies presented performing salpingoscopy that in infertile patient with endometriosis doesn't cause intraluminal abnormalities. ${ }^{6,7}$

Tubal infertile patients with hydrosalpinx have been identified as having significantly lower implantation and pregnancy rates than patients suffering from other types of tubal damage. ${ }^{8}$ Hydrosalpingeal fluid plays a causative role impairing implantation and/or embryo development. Any surgical intervention interrupting the communication between hydrosalpinx and uterus would obstruct the leakage of the hydrosalphingeal fluid and restore pregnancy rates. ${ }^{9}$

In the presence of unilateral hydrosalpinx and contralateral healthy tube, a unilateral salpingectomy can be suggested in order to achieve spontaneous conception. ${ }^{9}$ Proximal tubal ligation, salpingostomy and transvaginal aspiration may be considered as other surgical approaches. We performed salpingectomy to improve pregnancy rate in our patient.

In conclusion, in the presence of hydrosalpinx in infertile patients, although rare, intraluminal endometriosis should be kept in mind despite the absence of ovarian or peritoneal endometriotic foci. Minimally invasive procedures should be the treatment of choice in case of hydrosalpinx, which may arise from rare conditions that impair fertility.

\section{REFERENCES}

1.Rosai J, Sabin LH, Scully RE, Young RH, Clement PB. Tumors of the ovary, maldeveloped gonads, fallopian tube, and broad ligament. Atlas Tumor Pathol 1998; 160-161

2.Gruppos Italiano per lo Studio Dell'Endometriosi. Prevalence and anatomical distribution of endo metriosis in women with selected gynecological condition: results from a multicentric Italian study. Human Reprod 1994; 9:1158

3. Khoo JJ, Ismail MS, Tiu CC. Endometriosis of the appendix presenting as acute appendicitis.

Singapore Med J 2004; 45:435-6

4. Sait KH. Massive ascites as a presentation in a young woman with endometriosis: a case report. Fertil Steril 2008; 90:2015

5.Stock RJ. Postsalpingectomy endometriosis: a reas 
sessment. Obstet Gynecol 1982; 60:560-70

6. Heylen SM, Brosens IA, Puttemans PJ. Clinical value and cumulative pregnancy rates following rigid salpingoscopy during laparoscopy for infertil ity. Hum Reprod 1995; 10:2913-6

7.Bowman MC, Cooke ID. Comparison of fallopian tube intraluminal pathology as assessed by salpin goscopy with pelvic adhesions. Fertil Steril 1994; 61:464-9

8. Strandell A, Waldenström U, Nilsson L, Hamberger L. Hydrosalpinx reduces in vitro fertilization/em bryo transfer rates. Human Reprod 1994; 9:861-3

9. Gardner KD, Weissman A, Howles MC, Shoham Z eds. 3rd ed. Textbook of assisted reproductive techniques: Laboratory and clinical perspectives. New York: Informa, 2009:791-803 
